\title{
锗氮共掺碳化硅晶体杂质浓度表征及其电学性质研究
}

\author{
李 天 ${ }^{1,2}$, 陈秀芳 ${ }^{1,2}$, 杨祥龙 ${ }^{1,2}$, 谢雪健 ${ }^{1,2}$, 张福生 ${ }^{1,2}$, 肖龙飞 ${ }^{1,2}$, \\ 王荣塆 ${ }^{1,2}$, 徐现刚 ${ }^{1,2}$, 胡小波 ${ }^{1,2}$, 王瑞琪 ${ }^{2,3}$, 于 茫 ${ }^{2,3}$
}

(1. 山东大学 晶体材料国家重点实验室, 济南 250100; 2. 全球能源互联网(山东)协同创新中心, 济南 250061;

3. 国网山东省电力公司电力科学研究院，济南 250001)

摘 要: 采用物理气相传输 $(\mathrm{PVT})$ 法生长了 2 英寸 $(1$ 英寸 $=25.4 \mathrm{~mm})$ 锗氮 $(\mathrm{Ge}-\mathrm{N})$ 共掺和单一 $\mathrm{Ge}$ 掺杂碳化硅晶体材料, 并制备成 $10 \mathrm{~mm} \times 10 \mathrm{~mm}$ 的 $\mathrm{SiC}$ 晶片。利用半导体工艺技术在不同祄底的碳面上制备钛 $(\mathrm{Ti}) /$ 铂 $(\mathrm{Pt}) /$ 金 $(\mathrm{Au})$ 多层金属 电极。使用二次离子质谱仪(SIMS)、霍尔测试仪(Hall)等测试手段对其表征。结果表明, Ge 元素和 $\mathrm{N}$ 元素的共同掺 杂可以有效提高 $\mathrm{SiC}$ 中 $\mathrm{Ge}$ 元素的掺杂浓度, $\mathrm{Ge}$ 浓度可以达到 $1.19 \times 10^{19} / \mathrm{cm}^{3}$ 。所有晶片祄底均可以在不低于 $700^{\circ} \mathrm{C}$ 的 退火环境中形成欧姆接触, 且在 $700^{\circ} \mathrm{C}$ 时退火形成最佳欧姆接触。高浓度 $\mathrm{Ge}$ 掺杂祄底接触电阻明显小于低浓度 $\mathrm{Ge}$ 掺杂祄底接触电阻, 这表明可以通过提高晶体中 $\mathrm{Ge}$ 元素浓度来提高器件性能。Hall 测试结果表明, 随着 $\mathrm{Ge}$ 掺杂 浓度的升高, 祄底迁移率会逐渐降低。这是由于 $\mathrm{Ge}-\mathrm{N}$ 共掺后, $\mathrm{SiC}$ 晶格匹配度提高, $\mathrm{Ge}$ 元素的掺杂浓度变大, 增加 了杂质散射对迁移率的影响。

关 键 词: 物理气相传输法; Ge 掺杂; 晶格匹配; 欧姆接触; 迁移率

中图分类号: TB34 文献标识码: A

\section{Characterization and Electrical Property of Impurity Concentration in Ge-N Codoped SiC Crystals}

\author{
LI Tian ${ }^{1,2}$, CHEN Xiu-Fang ${ }^{1,2}$, YANG Xiang-Long ${ }^{1,2}$, XIE Xue-Jian $^{1,2}$, ZHANG Fu-Sheng $^{1,2}$, \\ XIAO Long-Fei ${ }^{1,2}$, WANG Rong-Kun ${ }^{1,2}$, XU Xian-Gang ${ }^{1,2}$, HU Xiao-Bo ${ }^{1,2}$, WANG Rui-Qi ${ }^{2,3}$, YU Peng ${ }^{2,3}$ \\ (1. State Key Laboratory of Crystal Materials, Shandong University, Jinan 250100, China; 2. Collaborative Innovation Center \\ for Global Energy Interconnection(Shandong), Jinan 250061, China; 3. State Grid Shandong Electric Power Research Institute, \\ Jinan 250001, China)
}

\begin{abstract}
Ge-N codoped and Ge doped $\mathrm{SiC}$ single crystals were grown by physical vapor transport (PVT) method. And the SiC ingots were fabricated into $10 \mathrm{~mm} \times 10 \mathrm{~mm} \mathrm{SiC}$ wafers for characterization. Semiconductor technology was used to fabricate $\mathrm{Ti} / \mathrm{Pt} / \mathrm{Au}$ metal contact on the carbide-terminated face of $\mathrm{SiC}$ wafers. Subsequently, all samples were characterized by secondary ion mass spectrometry (SIMS) and Hall measurements. The SIMS results showed that Ge-N codoping method could enhance the Ge doping concentration in SiC crystals effectively, which could achieve $1.19 \times 10^{19} / \mathrm{cm}^{3}$. According to Hall measurement, ohmic contact could be obtained when samples were
\end{abstract}

收稿日期: 2017-05-22; 收到修改稿日期：2017-09-04

基金项目: 国家重点研发计划(2016YFB0400401); 装备预研教育部联合基金(青年人才)(6141A0232); 国家自然科学基金 (51502156, 61327808, 61504075); 国家电网公司科技项目(SGSDDK00KJJS1600071); 国家重点基础研究发展计 划(973 计划)(2013CB632801)

National Key R\&D Program of China (2016YFB0400401); The joint Foundation of Equipment Development and State Education Ministry for Outstanding Researcher (6141A0232); National Natural Science Foundation of China (51502156, 61327808, 61504075); The Science and Technology Project of State Grid Corporation of China (SGSDDK00KJJS1600071); National Program on Key Basic Research Project (973 Program) (2013CB632801)

作者简介: 李 天(1992-), 男, 硕士研究生. E-mail: 13306490035@163.com

通讯作者: 陈秀芳, 教授.E-mail: cxf@sdu.edu.cn 
annealed at temperature higher than $700^{\circ} \mathrm{C}$ and the optimal annealing temperature was $700^{\circ} \mathrm{C}$. In addition, the contact resistance of the heavy Ge doped sample was lower than that of the light doped one, indicating that the ohmic contact property could be enhanced by improving the Ge doping concentrations in SiC crystals. Furthermore, as the increase of Ge doping concentration, the mobility gradually decreased. It was ascribed to that the Ge-N atoms matched well with $\mathrm{SiC}$ lattice in codoping method leading to higher Ge doping concentration. Under that condition, the impurity scattering effect became evident, which resulted in a lower mobility for Ge-N codoped sample.

Key words: Ge doping; lattice; Ohmic contact; mobility

$\mathrm{SiC}$ 作为第三代半导体材料, 与常见的 $\mathrm{Si}$ 和 GaAs 等半导体材料相比, 具有禁带宽度大、热导率 高、击穿电场强度大、载流子饱和迁移速度高等优 点, 在半导体照明、航空航天、电力电子、微波通 讯等高温电子以及高频大功率器件的领域里, $\mathrm{SiC}$ 是最富有应用前景的材料 ${ }^{[1-3]}$ 。

目前 $\mathrm{SiC}$ 掺杂研究工作的主要重心在 $\mathrm{n}$ 型杂质 ${ }^{[4-5]}$ 和 $\mathrm{p}$ 型杂质 ${ }^{[6-8]}$ 上, 较少关注 IV 族元素的掺杂。最 近, IV 族元素 $\mathrm{Ge}$ 在 $\mathrm{SiC}$ 中的掺杂开始受到关注 ${ }^{[9]}$, 这是由于 $\mathrm{Ge}$ 元素与 $\mathrm{Si}$ 和 $\mathrm{C}$ 元素具有相同的核外电 子数, 对于 $\mathrm{SiC}$ 的电子性能不会产生直接影响。Roe 等 ${ }^{[10]}$ 发现 $\mathrm{Ge}$ 元素可以降低 $\mathrm{SiC}$ 器件的接触电阻, 提 高迁移率。同时, 掺杂 $\mathrm{Ge}$ 会影响 $\mathrm{SiC}$ 晶体结构, 使

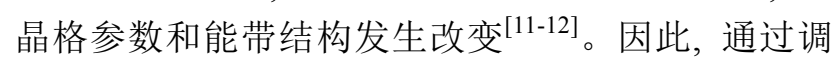
整 $\mathrm{Ge}$ 元素的掺杂量, 可以提高 $\mathrm{SiC}$ 器件的性能, 制 备性能优良的 $\mathrm{SiC}$ 器件。

制备 $\mathrm{Ge}$ 掺杂 $\mathrm{SiC}$ 器件, $\mathrm{SiC}$ 材料与金属电极之 间能否形成良好的欧姆接触是关键。目前, 已经有 许多金属可在 $\mathrm{SiC}$ 祄底上形成欧姆接触, 包括 $\mathrm{Ni}$ 、 $\mathrm{Ti} 、 \mathrm{Pt} 、 \mathrm{Al}$ 等 ${ }^{[13-18]}$ 。在这些金属中, $\mathrm{Ni}$ 和 $\mathrm{Ti}$ 是 $\mathrm{n}$ 型 $\mathrm{SiC}$ 欧姆接触中最常见的金属电极材料 ${ }^{[19]}$ 。为了更 好地利用不同金属电极在形成欧姆接触过程中的优 点, 电极制备一般使用多层金属。传统的用来形成 欧姆接触的金属电极层为 $\mathrm{Ti} / \mathrm{Al}$ 结构 ${ }^{[20-21]}, \mathrm{Ti}$ 和 $\mathrm{Al}$ 可以有效地降低祄底与金属之间的接触电阻, 但是 $\mathrm{Ti} / \mathrm{Al}$ 结构在退火过程中易氧化, 在 Al 层氧化形成 的 $\mathrm{Al}_{2} \mathrm{O}_{3}$ 会导致接触电阻增加, 从而影响欧姆接触 效果。为了避免温度过高导致 $\mathrm{Al}$ 层的氧化, 可以加 入一层金属 $\mathrm{Au}^{[22]}$, 使其具有较低的电阻率, 且不与 氧气发生反应。在 $\mathrm{Al}$ 层和 $\mathrm{Au}$ 层之间再加入一层金 属 $\mathrm{Pt}$, 可以有效增强欧姆接触的热稳定性 ${ }^{[23]}$ 。

Zhou 等 ${ }^{[24]}$ 报道在 $\mathrm{n}$ 型 $\mathrm{GaN}$ 上利用 $\mathrm{Ti} / \mathrm{Pt} / \mathrm{Au}$ 结 构形成欧姆接触, 在接触面形成了较低的接触电阻, 表明此金属结构可以获得良好的欧姆接触。目前此 结构在 $\mathrm{SiC}$ 祄底上的应用还较少。为了完全避免 $\mathrm{Al}$ 层氧化导致接触电阻增加, 本工作采用 $\mathrm{Ti} / \mathrm{Pt} / \mathrm{Au}$ 结
构形成欧姆接触。

\section{1 实验方法}

\section{1 样品的生长与制备}

采用物理气相传输( $\mathrm{PVT}$ )法生长 $\mathrm{SiC}$ 晶体, 使用 2 英寸 $(1$ 英寸 $=25.4 \mathrm{~mm})$ 正向 $6 \mathrm{H}-\mathrm{SiC}$ 硅面作为籽晶 面, 具体生长方法见参考文献 ${ }^{[25]}$ 。生长 $\mathrm{Ge}-\mathrm{N}$ 共掺 $\mathrm{SiC}$ 晶体, 掺杂剂为金属 $\mathrm{Ge}$ 粉(Alfa Aesar, 纯度 99.999\%), 将掺杂剂置于坩埚底部, 并与多晶粉料 混合。在晶体生长过程中向生长气氛中通入定量的 氮气, 生长温度为 $2100 \sim 2300{ }^{\circ} \mathrm{C}$, 生长压力为 5000 8000 Pa。对生长得到的晶锭进行标准的切割、 研磨和抛光后得到 $10 \mathrm{~mm} \times 10 \mathrm{~mm}$ 的 $\mathrm{SiC}$ 衬底, 厚 度为 $445 \mu \mathrm{m}$ 。使用电子束蒸发法在测试样品上制作 $1 \mathrm{~mm} \times 1 \mathrm{~mm}$ 的 $\mathrm{Ti} / \mathrm{Pt} / \mathrm{Au}$ 电极, 其中 $\mathrm{Ti} 、 \mathrm{Pt} 、 \mathrm{Au}$ 三种 金属的厚度分别为 $60 、 50$ 和 $600 \mathrm{~nm}$ 。

\section{2 样品表征}

采用 $\mathrm{EAG}$ 公司的二次离子质谱仪(SIMS)对样 品的掺杂浓度进行测试, SIMS 测试仪器精度可以达 到 ppm 甚至 ppb 的量级, 可以保证实验结果的准确 性。采用 Quatek Group 公司的 HL5500PC 型霍尔测 试仪测试带电极掺锗 $\mathrm{SiC}$ 样品的欧姆接触效果以及 样品迁移率随掺杂浓度的变化情况, 该测试仪的磁 场使用永磁体，磁场强度为 $0.33 \mathrm{~T}$ 。

\section{2 结果与讨论}

\subsection{Ge-N 共掺 SiC 晶体 Ge 浓度研究}

对 Ge-N 共掺的样品进行 SIMS 测试分析。图 1 为相同生长条件下, Ge-N 共掺样品和单一 Ge 掺杂 样品的 $\mathrm{Ge}$ 浓度对比曲线, 由图可以看出, 两种不同 生长方式得到的 $\mathrm{Ge}$ 掺杂浓度有较大的差别: Ge-N 共掺样品的 Ge 浓度可以达到 $1.19 \times 10^{19} / \mathrm{cm}^{3}$, 单一 $\mathrm{Ge}$ 掺杂样品的 $\mathrm{Ge}$ 浓度为 $2.52 \times 10^{18} / \mathrm{cm}^{3}$, 此浓度为 目前获得单一 $\mathrm{Ge}$ 掺杂 $\mathrm{SiC}$ 形成单晶的极限浓度, 继 


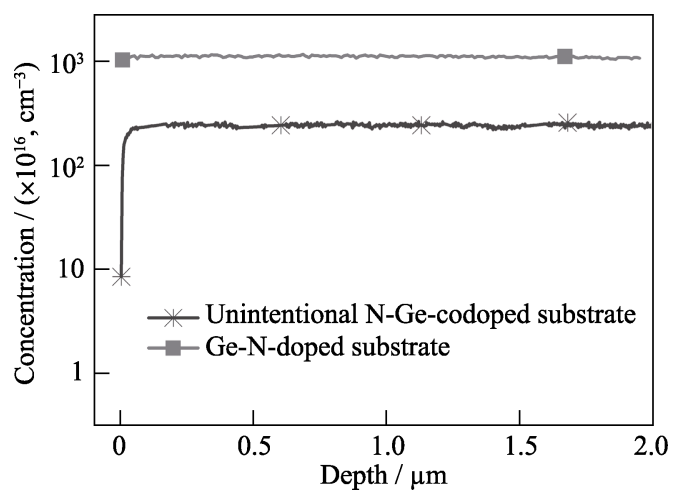

图 $1 \mathrm{Ge}-\mathrm{N}$ 共掺 $\mathrm{SiC}$ 祄底和单一 $\mathrm{Ge}$ 掺杂 $\mathrm{SiC}$ 祄底的 SIMS 测试结果

Fig. 1 SIMS analysis of Ge-N-codoped SiC substrate and Ge-doped SiC crystal substrate

续增大掺杂量会产生多晶。很明显, Ge-N 共掺样品 中 $\mathrm{Ge}$ 的浓度比单一 $\mathrm{Ge}$ 掺杂样品高出约一个数量 级。这是由于掺入的 $\mathrm{N}$ 原子会取代 $\mathrm{C}$ 原子 ${ }^{[26]}, \mathrm{N}$ 原 子的原子半径 $(0.075 \mathrm{~nm})$ 小于 $\mathrm{C}$ 原子的原子半径 $(0.086 \mathrm{~nm})$, 会引起晶胞参数的减小; 而掺入 $\mathrm{Ge}$ 原 子会取代 $\mathrm{Si}$ 原子 ${ }^{[9]}, \mathrm{Ge}$ 原子的原子半径 $(0.152 \mathrm{~nm})$ 大于 $\mathrm{Si}$ 的原子半径 $(0.146 \mathrm{~nm})$, 会引起晶胞参数的 增大。同时掺入 $\mathrm{N}$ 原子和 $\mathrm{Ge}$ 原子, 可以使 $\mathrm{SiC}$ 晶 体的晶格匹配度提高, 促进两种原子的掺杂。所以, 当两种元素同时掺杂时, 可以提高 $\mathrm{Ge}$ 元素在 $\mathrm{SiC}$ 晶 体中的掺杂浓度。

\section{$2.2 \mathrm{Ge}-\mathrm{N}$ 共掺 $\mathrm{SiC}$ 晶体欧姆接触研究}

为了形成理想的金属-半导体接触, 需要降低 金属功函数, 从而减小势垒高度, 形成欧姆接触。本 实验形成欧姆接触的电极材料为 $\mathrm{Ti} / \mathrm{Pt} / \mathrm{Au}$ 金属层, 祄底为不同 $\mathrm{Ge}$ 掺杂浓度 $\mathrm{SiC}$ 晶体, 在不同 $\mathrm{SiC}$ 祄底 的碳面上制备 $\mathrm{Ti} / \mathrm{Pt} / \mathrm{Au}$ 电极。在 $\mathrm{C}$ 面制备电极后, 进 行快速退火后会产生 $\mathrm{TiC}$ 。这是由于在退火过程中, $\mathrm{SiC}$ 中的 $\mathrm{C}$ 原子迁移到晶体表面与 $\mathrm{Ti}$ 金属进行反应, 形成了 $\mathrm{TiC} 。 \mathrm{C}$ 原子的扩散使 $\mathrm{SiC}$ 晶格中形成 $\mathrm{C}$ 空 位, 增加的 $\mathrm{C}$ 空位在 $\mathrm{Ti} / \mathrm{Pt} / \mathrm{Au}-\mathrm{SiC}$ 欧姆接触形成过 程中作为施主电子, 会降低祄底与金属之间的接触
电阻 ${ }^{[27]}$ 。所有样品的金属电极均制备在 C 面, 并进 行快速退火实验, 条件为 $600^{\circ} \mathrm{C} 、 700^{\circ} \mathrm{C} 、 800^{\circ} \mathrm{C}$ 的 $\mathrm{Ar}$ 气环境，持续时间为 $5 \mathrm{~min}$ 。不同样品的 $\mathrm{Ge}$ 掺杂 浓度如表 1 所示。样品 $\mathrm{A}$ 为相同生长条件下未掺杂 $\mathrm{Ge}$ 元素的 $\mathrm{SiC}$ 祄底材料。

表 1 不同 Ge 掺杂浓度的 $\mathrm{SiC}$ 样品

Table 1 SiC crystal samples with different Ge-doping concentrations

\begin{tabular}{ccc}
\hline Sample & Structure & $\begin{array}{c}\text { Ge doping } \\
\text { concentration } / \mathrm{cm}^{-3}\end{array}$ \\
\hline $\mathrm{A}$ & $\mathrm{Ti} / \mathrm{Pt} / \mathrm{Au} / \mathrm{SiC}$ & Undoped \\
$\mathrm{B}$ & $\mathrm{Ti} / \mathrm{Pt} / \mathrm{Au} / \mathrm{Ge}-\mathrm{SiC}$ & $\sim 5 \times 10^{17}$ \\
$\mathrm{C}$ & $\mathrm{Ti} / \mathrm{Pt} / \mathrm{Au} / \mathrm{Ge}-\mathrm{SiC}$ & $9.39 \times 10^{18}$ \\
$\mathrm{D}$ & $\mathrm{Ti} / \mathrm{Pt} / \mathrm{Au} / \mathrm{Ge}-\mathrm{SiC}$ & $1.19 \times 10^{19}$ \\
\hline
\end{tabular}

图 2 是分别在 $600^{\circ} \mathrm{C} 、 700^{\circ} \mathrm{C} 、 800^{\circ} \mathrm{C}$ 退火后样品 $\mathrm{C}$ 的伏安特性曲线图。从图 2(a)可以看出, 在 $600^{\circ} \mathrm{C}$ 退 火后样品 $\mathrm{C}$ 伏安特性曲线为非线性, 说明样品 $\mathrm{C}$ 经 $600^{\circ} \mathrm{C}$ 退火没有形成欧姆接触。如图 2(b) (c)所示, 样品 $\mathrm{C}$ 在 $700^{\circ} \mathrm{C}$ 以及 $800^{\circ} \mathrm{C}$ 退火后, 伏安特性曲线均 为线性, 说明此时形成了欧姆接触。这是因为随着 退火温度的升高, 在金属与半导体接触区域会出现 石墨化过程, 在祄底表面形成高质量的 $\mathrm{C}$ 悬空键, 降低了肖特基势垒高度, 从而形成欧姆接触 ${ }^{[19]}$ 。通 过对比图 2(b) (c) 发现, 样品 $\mathrm{C}$ 经 $700^{\circ} \mathrm{C}$ 退火接触电阻 小于 $800^{\circ} \mathrm{C}$ 退火, 形成的欧姆接触最好。对于样品 $\mathrm{A}$ 、 $\mathrm{B} 、 \mathrm{D}$, 不同温度退火后的伏安特性曲线和样品 $\mathrm{C}$ 有 相同的规律。以上结果可以表明, 对于 $\mathrm{Ge}-\mathrm{N}$ 共掺的 $\mathrm{SiC}$ 祄底, 同未掺 $\mathrm{Ge}$ 的 $\mathrm{SiC}$ 祄底具有相似的欧姆接 触形成规律, 形成欧姆接触的退火温度不低于 $700^{\circ} \mathrm{C}$, 且在 $700^{\circ} \mathrm{C}$ 退火可以形成最佳的欧姆接触效果。

图 3 是所有祄底在 $700^{\circ} \mathrm{C}$ 退火后的伏安特性曲 线图, 从实验结果显示的曲线斜率来看, 样品 $\mathrm{A}$ 的 伏安特性曲线斜率最大, 表明其欧姆接触最佳。这 是由于样品 $\mathrm{B} 、 \mathrm{C} 、 \mathrm{D}$ 生长过程中掺杂了 $\mathrm{Ge}$ 元素, 造 成晶体缺陷较多, 结晶质量较差 ${ }^{[9]}$ 。而对于 $\mathrm{Ge}-\mathrm{N}$ 共 掺的 $\mathrm{SiC}$ 晶体祄底来说, $\mathrm{Ge}$ 掺杂浓度较高的样品 $\mathrm{C}$

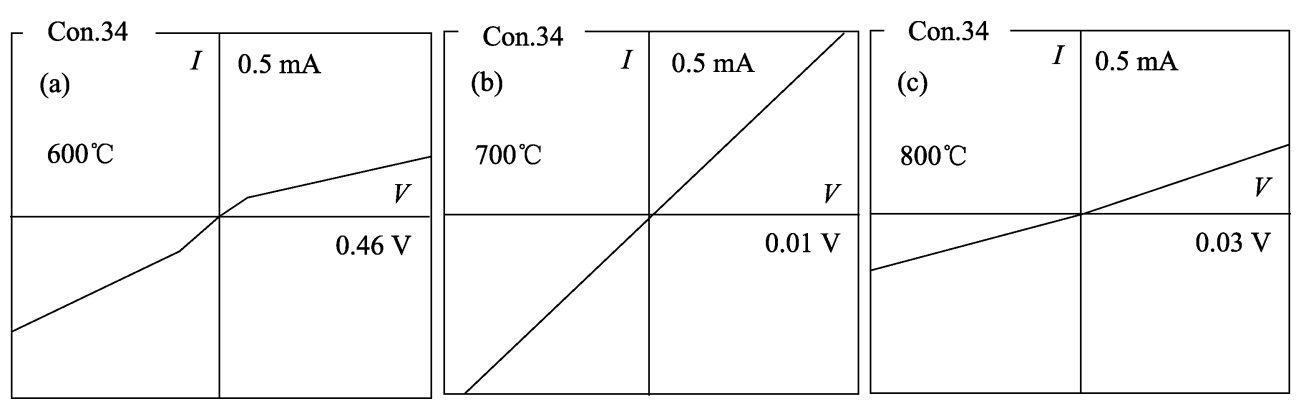

图 2 样品 $\mathrm{C}$ 在不同温度退火后的伏安特性曲线图

Fig. $2 I-V$ curves of sample $\mathrm{C}$ annealed at different temperatures 
和样品 $\mathrm{D}$ 的伏安特性曲线斜率明显大于 $\mathrm{Ge}$ 掺杂浓 度较低的样品 $\mathrm{B}$ 的伏安特性曲线斜率, 这表明, $\mathrm{Ge}$ 掺杂浓度大小对欧姆接触的形成会产生影响, 且 $\mathrm{Ge}$ 浓度越高, 形成的欧姆接触越好。这是因为 $\mathrm{Ge}$ 掺杂 浓度的增加会造成 $\mathrm{SiC}$ 晶格中 $\mathrm{Si}$ 原子向外扩散, 增 加的 $\mathrm{Si}$ 空位在 $\mathrm{Ti} / \mathrm{Pt} / \mathrm{Au}-\mathrm{SiC}$ 欧姆接触形成过程中作 为受主电子, 会降低祄底与金属之间的接触电阻, 对于欧姆接触的提高产生重要的作用 ${ }^{[28]}$ 。对于 $\mathrm{Ge}$ 掺杂浓度较高的样品 $\mathrm{C}$ 和样品 $\mathrm{D}$, 图中伏安特性曲 线斜率相差不大, 说明 $\mathrm{Ge}$ 浓度较高时形成的接触 电阻相差不大, 欧姆接触效果会达到某个极限。

\section{$2.3 \mathrm{Ge}-\mathrm{N}$ 共掺 $\mathrm{SiC}$ 晶体迁移率研究}

对 $700^{\circ} \mathrm{C}$ 退火后形成欧姆接触的样品 A、B、C、 $\mathrm{D}$ 进行霍尔测试分析。图 4 为不同样品迁移率变化 规律曲线。通过图 4 可以看到, 随着样品中 $\mathrm{Ge}$ 掺杂 浓度的升高, 迁移率数值依次降低。依据测试结果 分析, $\mathrm{Ge}-\mathrm{N}$ 共掺后, $\mathrm{SiC}$ 晶格匹配度提高, $\mathrm{Ge}$ 元素的 掺杂浓度增大, 增加了杂质散射对迁移率的影响, 最终导致迁移率降低。

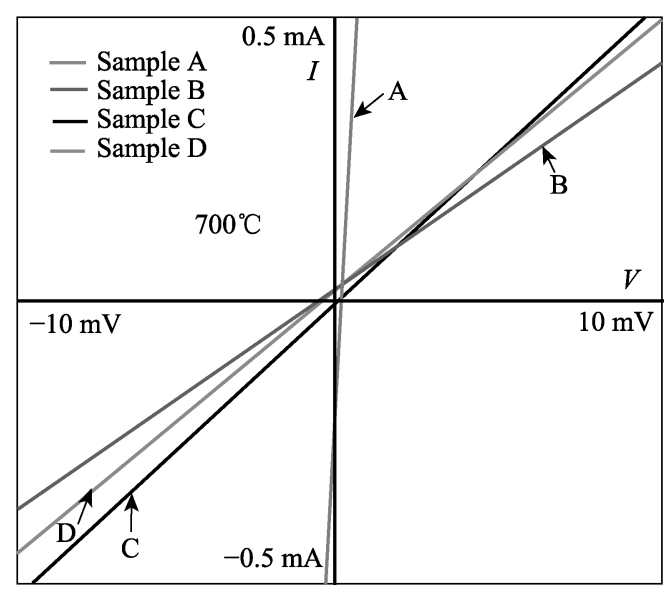

图 3 不同样品在 $700^{\circ} \mathrm{C}$ 退火后的伏安特性曲线图

Fig. $3 I-V$ curves of different samples annealed at $700^{\circ} \mathrm{C}$

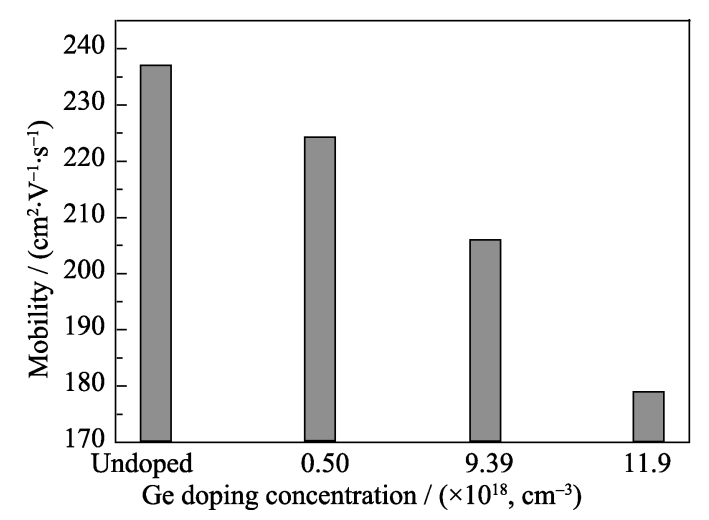

图 4 不同 $\mathrm{Ge}$ 掺杂浓度的 $\mathrm{SiC}$ 样品的迁移率变化规律曲线图 Fig. 4 Mobility curve of different Ge-doping concentration $\mathrm{SiC}$ crystal samples

\section{3 结论}

采用 PVT 法生长出 $\mathrm{Ge}-\mathrm{N}$ 共掺 $\mathrm{SiC}$ 晶体, 并研 究了其欧姆接触的形成规律和迁移率随 Ge 掺杂浓 度的变化规律。其中 $\mathrm{Ge}-\mathrm{N}$ 共掺 $\mathrm{SiC}$ 晶体的 $\mathrm{Ge}$ 掺杂 浓度达到 $1.19 \times 10^{19} / \mathrm{cm}^{3}, \mathrm{Ge}$ 浓度比单一 $\mathrm{Ge}$ 掺杂 $\mathrm{SiC}$ 晶体高出约一个数量级。在 $10 \mathrm{~mm} \times 10 \mathrm{~mm}$ 的不同 $\mathrm{Ge}-\mathrm{N}$ 共掺 $\mathrm{SiC}$ 祄底上制作了 $\mathrm{Ti} / \mathrm{Pt} / \mathrm{Au}$ 多层金属电极, 并在不同温度下进行退火, 在 $700^{\circ} \mathrm{C}$ 时形成了最佳 的欧姆接触。对于掺杂 $\mathrm{Ge}$ 元素的样品来说, 高掺杂 浓度的样品对形成良好的欧姆接触有很大帮助。通 过霍尔测试分析了 $\mathrm{Ge}-\mathrm{N}$ 共掺 $\mathrm{SiC}$ 晶体中迁移率的 变化规律, 发现随着 $\mathrm{Ge}$ 元素掺杂浓度的增加, 迁移 率减小。经过分析认为, $\mathrm{Ge}-\mathrm{N}$ 共掺后, $\mathrm{SiC}$ 晶格匹配 度提高, $\mathrm{Ge}$ 元素的掺杂浓度变大, 增加了杂质散射, 最终导致迁移率降低。

\section{参考文献:}

[1] CASADY J B, JOHNSON R W. Status of silicon carbide ( $\mathrm{SiC}$ ) as a wide-bandgap semiconductor for high-temperature applications: a review. Solid-State Electronics, 1996, 39(10): 1409-1422.

[2] ITOH A, MATSUNAMI H. Single crystal growth of SiC and electronic devices. Critical Reviews in Solid State and Material Sciences, 1997, 22(2): 111-197.

[3] BHATNAGAR M, BALIGA B J. Comparison of 6H-SiC, 3C-SiC, and Si for power devices. IEEE Transactions on Electron Devices, 1993, 40(3): 645-655.

[4] ROST H J, DOERSCHEL J, IRMSCHER K, et al. Influence of nitrogen doping on the properties of $4 \mathrm{H}-\mathrm{SiC}$ single crystals grown by physical vapor transport. Journal of Crystal Growth, 2003, 257(1): 75-83.

[5] UEMOTOU T. Reduction of ohmic contact resistance on n-type $6 \mathrm{H}-\mathrm{SiC}$ by heavy doping. Japanese Journal of Applied Physics, 1995, 34(1A): L7.

[6] STRAUBINGER T L, BICKERMANN M, WEINGÄRTNER R, et al. Aluminum p-type doping of silicon carbide crystals using a modified physical vapor transport growth method. Journal of Crystal Growth, 2002, 240(1): 117-123.

[7] MÜLLER R, KÜNECKE U, WEINGÄRTNER R, et al. High Al-doping of SiC using a modified PVT (M-PVT) growth set-up. Materials Science Forum. Trans. Tech. Publications, 2005, 483: $31-34$.

[8] BICKERMANN M, HOFMANN D, RASP M, et al. Study of boron incorporation during PVT growth of p-type $\mathrm{SiC}$ crystals. Materials Science Forum. Trans Tech Publications, 2001, 353: 49-52.

[9] ZHANG FU-SHENG, CHEN XIU-FANG, CUI YING-XIN, et al. Defects in Ge doped SiC crystals. Journal of Inorganic Materials, 2016, 31(11): 1166-1170.

[10] ROE K J, DASHIELL M W, XUAN G, et al. Ge incorporation in $\mathrm{SiC}$ and the effects on device performance. High Performance Devices, 2002. Proceedings. IEEE Lester Eastman Conference on. IEEE, 2002: 201-206.

[11] GHOSH A, VARADACHARI C. Theoretical derivations of a direct band gap semiconductor of $\mathrm{SiC}$ doped with Ge. Journal of Electronic Materials, 2015, 44(1): 167.

[12] ZANG YUAN, CAO LIN, LI LIAN-BI, et al. Theoretical study of 
electrical and optical properties of Ge-doped 6H-SiC. Laser \& Optoelectronics Progress, 2015, 52(6): 209-215.

[13] CHEN J S, BACHLI A, NICOLET M A, et al. Contact resistivity of Re, Pt and Ta films on n-type $\beta$-SiC: preliminary results. Materials Science and Engineering: B, 1995, 29(1/2/3): 185-189.

[14] KONISHI R, YASUKOCHI R, NAKATSUKA O, et al. Development of $\mathrm{Ni} / \mathrm{Al}$ and $\mathrm{Ni} / \mathrm{Ti} / \mathrm{Al}$ ohmic contact materials for p-type 4H-SiC. Materials Science and Engineering: B, 2003, 98(3): 286-293.

[15] OHYANAGI T, ONOSE Y, WATANABE A. Ti/Ni bilayer Ohmic contact on $4 \mathrm{H}-\mathrm{Si}$ C. Journal of Vacuum Science \& Technology B: Microelectronics and Nanometer Structures Processing, Measurement, and Phenomena, 2008, 26(4): 1359-1362.

[16] CHONG-CHONG D, XUE-CHAO L, TIAN-YU Z, et al. Effects of annealing temperature on the electrical property and microstructure of aluminum contact on $\mathrm{n}$-type 3C-SiC. Chinese Physics B, 2014, 23(6): 066803.

[17] DAI C C, LIU X C, ZHOU T Y, et al. Effect of annealing temperature on the contact properties of $\mathrm{Ni} / \mathrm{V} / 4 \mathrm{H}-\mathrm{SiC}$ structure. AIP Advances, 2014, 4(4): 047125.

[18] TIAN-YU Z, XUE-CHAO L, WEI H, et al. Electrical properties and microstructural characterization of $\mathrm{Ni} / \mathrm{Ta}$ contacts to $\mathrm{n}$-type 6H-SiC. Chinese Physics B, 2015, 24(12): 126801.

[19] ZHOU T Y, LIU X C, DAI C C, et al. Effect of graphite related interfacial microstructure created by high temperature annealing on the contact properties of $\mathrm{Ni} / \mathrm{Ti} / 6 \mathrm{H}-\mathrm{SiC}$. Materials Science and Engineering: B, 2014, 188: 59-65.
[20] CROFTON J, BARNES P A, WILLIAMS J R, et al. Contact resistance measurements on p-type 6H-SiC. Applied Physics Letters, 1993, 62(4): 384-386.

[21] CROFTON J, BEYER L, WILLIAMS J R, et al. Titanium and aluminum-titanium ohmic contacts to p-type SiC. Solid-State Electronics, 1997, 41(11): 1725-1729.

[22] CHANG S C, WANG S J, UANG K M, et al. Investigation of $\mathrm{Au} / \mathrm{Ti} / \mathrm{Al}$ ohmic contact to N-type $4 \mathrm{H}-\mathrm{SiC}$. Solid-state electronics, 2005, 49(12): 1937-1941.

[23] LEE C T, KAO H W. Long-term thermal stability of Ti/Al/Pt/Au Ohmic contacts to n-type GaN. Applied Physics Letters, 2000, 76(17): 2364-2366.

[24] ZHOU L, LANFORD W, PING A T, et al. Low resistance Ti/Pt/Au ohmic contacts to p-type GaN. Applied Physics Letters, 2000, 76(23): 3451-3453.

[25] YANG KUN, CHEN XIU-FANG, YANG XIANG-LONG, et al. Growth of high purity semi-insulting $4 \mathrm{H}-\mathrm{SiC}$ single crystals. Journal of Synthetic Crystals, 2014, 43(11): 3055-3057.

[26] LARKIN D J, NEUDECK P G, POWELL J A, et al. Site-competition epitaxy for superior silicon carbide electronics. Applied Physics Letters, 1994, 65(13): 1659-1661.

[27] XU M, HU X, PENG Y, et al. Fabrication of ohmic contact on the carbon-terminated surface of n-type silicon carbide. Journal of Alloys and Compounds, 2013, 550: 46-49.

[28] JOHNSON B J, CAPANO M A. Mechanism of ohmic behavior of $\mathrm{Al} / \mathrm{Ti}$ contacts to p-type $4 \mathrm{H}-\mathrm{SiC}$ after annealing. Journal of Applied Physics, 2004, 95(10): 5616-5620. 